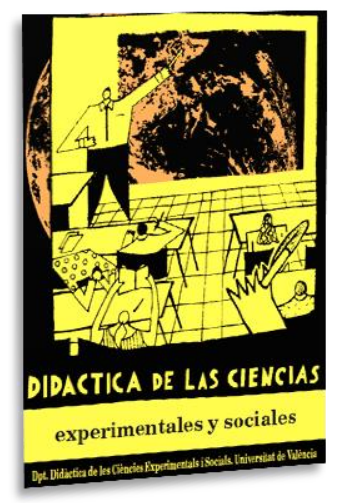

\title{
Territorios epistémicos en la práctica pedagógica en ciencias sociales
}

\section{Epistemic territories in pedagogical practice in social sciences}

DOI: $10.7203 / D C E S .35 .12913$

\author{
Liliana Angélica Rodríguez Pizzinato \\ Universidad Distrital Francisco José de Caldas (Colombia) \\ larodriguezp@udistrital.edu.co
}

\begin{abstract}
RESUMEN: Los resultados discutidos en el artículo forman parte de la investigación Territorios epistémicos y práctica pedagógica en las licenciaturas de biología y ciencias sociales de la Universidad Distrital Francisco José de Caldas (Colombia), en la línea de investigación Enseñanza de las ciencias, contexto y diversidad cultural del Doctorado Interinstitucional en Educación que se está cursando en Colombia. En este trabajo se pregunta por los territorios epistémicos que configuran los estudiantes de la licenciatura en ciencias sociales, sobre la práctica pedagógica que desarrollan. Se adopta la categoría de territorio epistémico para comprender la práctica docente, más allá de la aplicación en la escuela de la teoría abordada en la universidad, para ubicarla como una actividad que se produce en la intersección entre saberes $\mathrm{y}$ acciones del futuro docente. El proceso metodológico se fundamenta en una perspectiva cualitativa interpretativa y adopta el estudio de caso como sistema delimitado de la realidad a investigar.
\end{abstract}

Palabras ClaVe: práctica pedagógica, formación inicial docente, territorios epistémicos, narrativa, conglomerados de relevancia.

\begin{abstract}
The results discussed in the article are part of the research Epistemic territories and pedagogical practice in the bachelor degrees in biology and social sciences of the Francisco José de Caldas District University, in the line of the research Teaching of sciences, context and cultural diversity of the interinstitutional doctorate in education that is being studied in Colombia. This paper approaches the epistemic territories that set up the students of the bachelor's degree in social sciences, about the pedagogical practice they develop. The category of epistemic territory is adopted to understand the teaching practice, beyond the application in the school of the theory approached in the university, to locate it like an activity that occurs in the intersection between knowledges and actions of the future teacher. The methodological process is based on an interpretative qualitative perspective and adopts the case study as a delimited system of reality to investigate.
\end{abstract}

KEYWORDS: pedagogical practice, initial teacher training, epistemic territories, narrative, conglomerates of relevance.

Fecha de recepción: julio de 2018

Fecha de aceptación: noviembre de 2018

Investigación doctoral desarrollada en el Doctorado Interinstitucional en Educación de la Universidad Distrital Francisco José de Caldas y orientada por la Dra. Adela Molina Andrade. La autora es miembro del Grupo de Investigación INTERCITEC. 


\section{INTRODUCCIÓN}

El trabajo se ubica en la línea de formación de profesores, cuyos resultados en investigación educativa muestran una transformación de los paradigmas que miran al docente desde la racionalidad técnica como instructor y transmisor de conocimiento; hacia paradigmas que lo asumen como un sujeto reflexivo y reconocen a nivel pedadógigo, la validez de su pensamiento, ya que tiene concepciones, emite juicios, toma decisiones, genera rutinas y produce un conocimiento práctico propio sobre desarrollo profesional, como afirma Mellado y González (2000).

Por otra parte, tiene en cuenta la práctica docente desarrollada en su etapa de formación inicial, caracterizada por considerar la existencia de una concepción pedagógica personal que se constituye de forma natural y no producto de la reflexión de las propias experiencias escolares; por lo cual esta concepción es más estable, al estar ligada al sistema de creencias del futuro licenciado. También supone la presencia de un conocimiento científico, que le permite al docente transformarlo en representaciones comprensibles para los procesos de enseñanza y aprendizaje.

Así, el artículo presenta algunos resultados de la tesis doctoral, que propone como problema de investigación la comprensión de los territorios epistémicos que los estudiantes de la licenciatura en educación básica con énfasis en ciencias sociales (LEBECS) configuran de su práctica pedagógica. Esta investigación es cualitativa interpretativa con carácter etnográfico e incorpora según Ameigeiras (2009), una estrategia de abordaje de la realidad que permite cambiar la forma de construcción del conocimiento en la práctica social, con un compromiso fundamental del investigador en su trabajo de campo y en su relación con los actores sociales.

Por su parte, el diseño de investigación es dinámico desde la postura de Maxwell (1996), en el cual los dominios teórico, metódico y analítico se encuentran interconectados, dado el carácter cíclico, abierto, flexible y reflexivo de este tipo de diseño. De igual manera, la comprensión de la relación entre conocimientos y acciones de los profesores en formación inicial que permiten identificar los territorios epistémicos que estos constituyen, asume la modalidad de estudio intrínseco de caso que en palabras de Stake (1999), busca aprender sobre un caso particular y no estudiarlo para que se aplique a otros.

Por esta razón, el estudio de caso presenta una narrativa no de sujetos individuales e independientes, sino de situaciones pedagógicas en las que se muestran facetas convergentes y/o divergentes en su acción, cuyas decisiones plantean diferencias según Barrón (2015), entre el docente experto y el docente novel, ya que el primero toma decisiones basado en su experiencia, conocimiento de la disciplina y reflexiones sobre su práctica y el segundo, se dedica a pensar más en la resolución de problemas de la enseñanza, que en las razones y momentos de producción de los mismos.

En el contexto de formación inicial docente, la importancia de la investigación se expresa por una parte, en la elaboración de una propuesta de orientaciones para la licenciatura de referencia, así como para la Facultad de Ciencias y Educación donde esta funciona, en relación con la organización de una práctica pedagógica colectiva, conjunta y compartida entre escuela y universidad. Por otra, en la proyección de criterios para el diseño de una política educativa de formación inicial docente, basada en el descubrimiento de sus propias teorías implícitas, que aportan explicaciones en la organización de la práctica docente. Así como, en el reconocimiento del sentido de su propio trabajo y de los procesos internos que activa el futuro licenciado, especialmente cuando planifica e interviene en el aula.

De acuerdo con lo anterior, el artículo se organiza en cuatro partes: la primera presenta una conceptualización general de las categorías centrales de la investigación, la segunda una descripción del procesamiento de información, la tercera aborda los resultados más significativos de la interpretación de los datos y en la cuarta se discuten las conclusiones sobre algunos territorios epistémicos identificados. 


\section{CATEgorías CENTRALES DE LA INVESTIGACIÓN}

\subsection{Práctica pedagógica y formación inicial docente}

La práctica pedagógica estudiada es la realizada en la etapa de formación inicial docente, la cual se considera como un proceso institucionalizado (Jessup et al., 2013), en el que los programas, proyectos y/o prácticas generadas, se dan en el marco de una intencionalidad de profesionalización y/o preparación para el ejercicio laboral, que se concreta en la obtención de un título universitario. Así, la formación inicial implica un proceso individual y social, en el cual, el futuro docente incorpora experiencias y saberes que recoge en los contextos personales y escolares de formación.

En este sentido, los estudios sobre concepciones epistemológicas que los docentes manifiestan en el proceso de enseñanza, se orientan como afirma Barrón (2015) en la búsqueda de resultados eficaces en el aprendizaje de los estudiantes. Dichos estudios, se han realizado desde distintas perspectivas que van desde el paradigma proceso-producto hasta el crítico e interpretativo. También hay una perspectiva de racionalidad práctica vinculada con la visión de un docente reflexivo, que como señala Fenstermacher (1994), piensa la construcción de conocimiento en la enseñanza, destacando las explicaciones del papel del docente en la elaboración del conocimiento sobre su práctica.

Esta perspectiva avanza respecto a las anteriores, al reconocer el valor de un conocimiento práctico y personal, presente en las representaciones implícitas del docente acerca de la enseñanza, el aprendizaje, el currículo y/o el estudiante, que permite comprender relaciones entre conocimiento y acción. Los estudios de Claxton y Murrell (1987), se refieren al carácter sistémico y coherente del pensamiento del profesor y lo denominan teorías personales docentes, las cuales tienen relación con el planteamiento de teorías implícitas, propuesto por Rodrigo, Rodríguez y Marrero (1993), quienes afirman que los docentes en formación manifiestan conjuntos coherentes de ideas, preconcepciones y creencias sobre sus procesos formativos y la toma de decisiones en su práctica docente.

Estas teorías implícitas se equiparan también con un conocimiento ingenuo (Hatano \& Inagaki 1994; y Keil, 1992), duradero, aplicable, funcional y fácilmente recuperable en la acción y el conocimiento; lo cual dificulta su posibilidad de modificación. Lo anterior, aporta un contenido explicativo para comprender situaciones que se producen cotidianamente en el aula, a partir de modelos implícitos que infieren los actores educativos, producto de su experiencia escolar sobre lo que es y debe ser la enseñanza y el aprendizaje.

\subsection{Territorios epistémicos}

La referencia al territorio se realiza como analogía, es decir, como un recurso cognitivo que favorece comparaciones de un concepto de un área de conocimiento en otra, cuya perspectiva vincula esta categoría con los campos epistemológico y geográfico. En el primer caso, se fundamenta en el reconocimiento de la pluralidad y heterogeneidad de conocimientos, en los cuales el científico forma parte de ellos, no con un carácter exclusivo como la ciencia moderna le ha dado.

Lo anterior, se plantea en la perspectiva de la ecología de saberes propuesta por De Sousa Santos (2010), en la que la autonomía de los conocimientos no se ve comprometida por las interconexiones que se presentan entre ellos. La ecología de saberes propone un reconocimiento de la diversidad epistemológica del mundo, avanzando en la idea de inter conocimiento, que permite aprender otros conocimientos sin olvidar el propio.

Con respecto a la analogía geográfica, Nates (2011) en su propuesta de territorialización del conocimiento, entiende que las clasificaciones que elaboran los sujetos les permiten intervenir el mundo practicado y vivido, es decir, que en los territorios epistémicos se funden conocimiento y acción. Por ello, estos se consideran espacios de interacción con una doble condición, en cuanto a 
los límites que se pueden establecer entre enfoques teórico-conceptuales, valores pedagógicos que se establecen y las fronteras que posibilitan la comunicación entre ellos.

En este sentido, los territorios epistémicos, no solo se constituyen con los campos de conocimiento propios de la profesión, sino con las actividades prácticas socialmente valoradas en las que la cognición distribuida en términos de Cole y Engrestrom (2001), aportan la visión de un conocimiento producido tanto en la mente de los sujetos, como en los procesos comunicativos y axiológicos que ellos desarrollan.

Los aspectos mencionados, son fundamentales de acuerdo con Rodríguez (2015), en la consideración de la emergencia de una categoría como la de territorios epistémicos, que asumiría la existencia de campos de conocimientos y acciones que se interconectan a nivel afectivo y cognitivo para hacer de la enseñanza un proceso más vigoroso y significativo.

\section{LECTURA DE LA INFORMACIÓN}

El enfoque de investigación reconoce el valor de los sentidos y significados expresados en diversas situaciones sociales para comprender el ámbito en el que se producen; se interesa de acuerdo con Rodríguez y Valldeoriola (2009) por la realidad tal y como la interpretan los sujetos, respetando el contexto donde dicha realidad social es construida; también reconoce el valor de los registros de observación realizados en el trabajo de campo, participando según Hammersley y Atkinson (1994), en los ámbitos escolares en los que desarrollan la práctica pedagógica los futuros docentes de la LEBECS.

Por otra parte, la investigación acude al estudio de caso desde la postura de Smith (1979) como un sistema acotado que en su condición de particularidad, ofrece posibilidades de comprensión sobre situaciones detalladas que en él se pueden encontrar. Para ello, se parte del criterio propuesto por Neiman y Quaranta (2009) de comparación analítica de similitud, para la definición de conclusiones a partir de la observación y comparación de elementos similares de una situación pedagógica.

La ruta metodológica de la investigación se sintetiza en la tabla 1, de la cual se destacan algunos rasgos del proceso de recolección de información desarrollado en la etapa exploratoria y de sistematización, así como de algunos resultados obtenidos en esta última.

TABLA 1. Desarrollo de la investigación

\begin{tabular}{|c|c|}
\hline Etapas & Acciones \\
\hline \multirow{5}{*}{ Exploratoria } & Reconocimiento de los escenarios formativos en la licenciatura. \\
\hline & Revisión documental sobre las categorías centrales de la investigación. \\
\hline & Construcción, validación y aplicación de los casos para el protocolo de entrevista. \\
\hline & $\begin{array}{l}\text { Aplicación del protocolo de entrevista para identificar y caracterizar los posibles participantes } \\
\text { (estudiantes, directores de práctica y profesores titulares en la institución escolar) que permitirían } \\
\text { la realización de la observación de clase. }\end{array}$ \\
\hline & Diseño y validación del protocolo de observación. \\
\hline \multirow{7}{*}{ Sistematización } & Definición de categorías metodológicas para orientar la interpretación de información. \\
\hline & Diligenciamiento del consentimiento informado. \\
\hline & $\begin{array}{l}\text { Reconocimiento de algunos aspectos de la vida personal y escolar de los participantes en la } \\
\text { investigación, que se registran en el diario de campo de la investigadora. }\end{array}$ \\
\hline & $\begin{array}{l}\text { Aplicación del protocolo de observación en las clases de los participantes que aceptan vincularse } \\
\text { al ejercicio de investigación y elaboración de narrativas producto del proceso mencionado } \\
\text { anteriormente. }\end{array}$ \\
\hline & Procesamiento e interpretación de la información con el software ATLAS-Ti. \\
\hline & Validación de resultados (participantes y expertos). \\
\hline & Contrastación de los resultados obtenidos con otras investigaciones. \\
\hline
\end{tabular}

Fuente: Elaboración propia 
En cuanto a la recolección de información esta se inicia en la etapa exploratoria con la aplicación de un protocolo de entrevista para identificar los participantes en la investigación. Para ello, se adopta el muestreo intencional propuesto por Maxwell (1996), en el cual escenarios, sujetos y/o acontecimientos son escogidos deliberadamente. Así, se definen los procesos de práctica pedagógica, en un grado de básica primaria y otro de básica secundaria de una institución escolar pública de Bogotá, en la que participan los profesores en formación inicial de LEBECS y el director de práctica que orienta dicho proceso.

El acopio de información continua en la etapa de sistematización, con elaboración del diario de campo por parte de la investigadora, entendido como un recurso metodológico que permite de acuerdo con Porlán \& Martín (1994), interrogar la realidad de la práctica pedagógica, constituyéndose en una fuente de testimonio sobre los registros realizados, en diferentes momentos de diálogo con los participantes en la investigación, que aportan a su vez en la comprensión de las actuaciones que pueden circular en el aula.

Así mismo, se aplica el protocolo de observación en las clases de los participantes vinculados al proceso de investigación y la elaboración de narrativas producto del registro realizado, para profundizar en la comprensión de significados y puntos de vista que manifiestan sobre la práctica pedagógica. El ejercicio dialógico con los participantes, permite reconstruir las narrativas en un proceso de modificación, adición, omisión y/o complementación, aplicando los criterios de reflexividad y voces múltiples.

En el primer criterio, es posible pensar el rol del investigador como sujeto reflexivo, que hace conciencia de los efectos que tiene su participación en la interacción y reciprocidad con los participantes en la investigación. En el segundo criterio, los resultados no se expresan desde la voz unilateral del investigador, sino desde una construcción colectiva con los futuros docentes y/o directores de práctica de la licenciatura.

\subsection{Categorías de investigación}

Los enunciados de los participantes sobre su acción pedagógica en el aula, forman parte del relato presente en la narrativa y se constituyen en unidades de significado que manifiestan la intervención que realizan. Estas permiten descubrir algunas relaciones entre conocimientos y acciones frente a su práctica, cuya revisión constante en los datos permite resignificar las interpretaciones realizadas, transformando progresivamente la categorización en la investigación (Tabla 2).

Las súper familias, familias y códigos en el software ATLAS-Ti permiten la categorización de la información. Así, las súper familias agrupan sistemáticamente la información, aduciendo a nociones espaciales y epistemológicas que como analogías se expresan en lo cognitivo. En cuanto a las familias, estas corresponden a un conjunto de elementos que comparten una cualidad, relacionada con los grupos de códigos establecidos en la interpretación de los datos.

Los códigos se constituyen en unidades básicas de análisis, representados en palabras clave, indicadores de conceptos, expresiones y/o temas similares descubiertos. Para ello, se desarrolla un proceso de codificación de información combinando una forma deductiva y otra inductiva. La primera, implica según Maxwell (1996) la aplicación a los datos de unidades preestablecidas, para identificar niveles de recurrencia en las mismas y la segunda plantea de acuerdo con Creswell (1994), el surgimiento de unidades de manera múltiple y abierta, en función de lo que va apareciendo como relevante en los datos.

En la tabla 3, se presenta una selección aleatoria de unidades básicas de significado, asignadas a la información prescriptiva o inferencial compilada durante la investigación. 
TABLA 2. Síntesis categorización

\begin{tabular}{|c|c|c|c|}
\hline Superfamilias & Concepto & Familias & Concepto \\
\hline $\begin{array}{l}\text { Límites y } \\
\text { fronteras } \\
\text { epistémicas }\end{array}$ & $\begin{array}{l}\text { Límites como componentes fijos que } \\
\text { demarcan separación. } \\
\text { Fronteras como espacios dinámicos } \\
\text { de encuentro de múltiples órdenes. }\end{array}$ & $\begin{array}{l}\text { Ideas sobre las } \\
\text { disciplinas y } \\
\text { contenidos } \\
\text { escolares }\end{array}$ & $\begin{array}{l}\text { Relaciones con el conocimiento } \\
\text { disciplinar, experiencial, escolar, } \\
\text { académico y/o profesional y con el } \\
\text { ámbito de enseñanza a nivel } \\
\text { cognitivo, procedimental, } \\
\text { actitudinal y valorativo. }\end{array}$ \\
\hline $\begin{array}{l}\text { Territorialidad } \\
\text { es específicas }\end{array}$ & $\begin{array}{l}\text { Territorialidades de hegemónicas, } \\
\text { protagónicas, } \\
\text { privilegiadas y/o jerarquizadas en el } \\
\text { aula de clase. }\end{array}$ & $\begin{array}{l}\text { Ideas sobre la } \\
\text { profesión docente }\end{array}$ & $\begin{array}{l}\text { Relaciones sobre el ser docente y su } \\
\text { ejercicio profesional. }\end{array}$ \\
\hline $\begin{array}{l}\text { Fuente de } \\
\text { información }\end{array}$ & $\begin{array}{l}\text { Emisores de signos y señales que } \\
\text { pueden mostrar teorías implícitas en } \\
\text { los docentes en formación. }\end{array}$ & \multirow{2}{*}{$\begin{array}{l}\text { Ideas sobre el } \\
\text { saber pedagógico }\end{array}$} & \multirow{2}{*}{$\begin{array}{l}\text { Percepciones del futuro licenciado } \\
\text { sobre su actuación en la práctica y } \\
\text { el espacio escolar donde se } \\
\text { desenvuelve. }\end{array}$} \\
\hline Palimpsesto & $\begin{array}{l}\text { Muestra las huellas que permanecen } \\
\text { en las teorías implícitas de los } \\
\text { docentes en formación, a nivel de } \\
\text { creencias, ideas espontáneas, } \\
\text { tradicionales y/o académicas. }\end{array}$ & & \\
\hline
\end{tabular}

Fuente. Elaboración propia

TABLA 3. Síntesis categorización deductiva e inductiva

\begin{tabular}{|c|c|c|}
\hline Familia & Códigos deductivos & $\begin{array}{l}\text { Hace referencia a expresiones y/o actitudes del } \\
\text { practicante... }\end{array}$ \\
\hline $\begin{array}{l}\text { Ideas sobre las disciplinas y } \\
\text { contenidos escolares }\end{array}$ & $\begin{array}{l}\text { Enseñanza de } \\
\text { contenido }\end{array}$ & $\begin{array}{l}\text { Sobre la selección y forma de trabajo de los contenidos en el } \\
\text { aula. }\end{array}$ \\
\hline $\begin{array}{l}\text { Ideas sobre la profesión } \\
\text { docente }\end{array}$ & Manejo de poder & $\begin{array}{l}\text { Referidas al ejercicio de autoridad en clase, que puede tener } \\
\text { connotaciones horizontales y/o autoritarias y/o de autoridad } \\
\text { y/o de ninguna autoridad. }\end{array}$ \\
\hline $\begin{array}{l}\text { Ideas sobre el saber } \\
\text { pedagógico }\end{array}$ & $\begin{array}{l}\text { Percepción del } \\
\text { ambiente educativo }\end{array}$ & $\begin{array}{l}\text { Relacionada con el comportamiento que asumen sus } \\
\text { estudiantes ante la clase, las actividades y/o el practicante } \\
\text { mismo, así como del currículo y/o modelo pedagógico que } \\
\text { elija la institución escolar. }\end{array}$ \\
\hline Familia & Códigos emergentes & $\begin{array}{l}\text { Hace referencia a expresiones y/o actitudes del } \\
\text { practicante... }\end{array}$ \\
\hline $\begin{array}{l}\text { Ideas sobre las disciplinas y } \\
\text { contenidos escolares }\end{array}$ & $\begin{array}{l}\text { Relación otros } \\
\text { conocimientos: } \\
\text { escolar }\end{array}$ & $\begin{array}{l}\text { Sobre otros conocimientos a los que se hace referencia en el } \\
\text { desarrollo de un contenido (experiencial, escolar, } \\
\text { académico, profesional). }\end{array}$ \\
\hline $\begin{array}{l}\text { Ideas sobre la profesión } \\
\text { docente }\end{array}$ & Diálogo en el aula & $\begin{array}{l}\text { Relacionas con la interacción comunicativa entre los } \\
\text { estudiantes del curso, entre estudiante-practicante y entre } \\
\text { practicantes. }\end{array}$ \\
\hline $\begin{array}{l}\text { Ideas sobre el saber } \\
\text { pedagógico }\end{array}$ & Clase expositiva & $\begin{array}{l}\text { Referidas a la clase magistral dirigida especialmente por el } \\
\text { docente o aquellos momentos en los que los estudiantes } \\
\text { asumen este rol con sus compañeros. }\end{array}$ \\
\hline
\end{tabular}

Fuente. Elaboración propia 


\section{HALlaZgOS DE LA INVESTIGACIÓN}

\subsection{Anuncios en la narrativa}

La perspectiva de la narrativa está presente en diversas áreas de conocimiento, para la investigación se adopta el enfoque de Elbaz (1983, 1991) sobre su aplicación en educación (curriculum, vida de los docentes y la de los investigadores acerca de las otras dos). Así, el relato se elabora, con la participación de cuatro estudiantes de últimos semestres de la licenciatura y su director de práctica, quienes desarrollan su intervención en cuarto y séptimo grado del nivel básico de la Institución Educativa Distrital Toberín.

Dicho relato, se presenta a los participantes para leerlo, comentarlo, discutirlo y ajustarlo de acuerdo con la significación que estos otorgan a sus decisiones pedagógicas. Por ello, las reflexiones compartidas con los profesores en formación inicial, son fundamentales para comprender en sus teorías implícitas, como lo asumen Clark y Peterson (1990), las experiencias educativas que están viviendo y desde las cuales actúan racionalmente. Dichas teorías son apropiadas para dar regularidad a la experiencia que el docente va desarrollando y sirven de estructura intelectual en los procesos de enseñanza y aprendizaje a los cuales se enfrentan (Rando y Menges, 1991).

Así, las actuaciones de los profesores en formación inicial se organizan en cuatro tópicos considerados por ellos mismos, en la narrativa como los más relevantes, con sus respectivas nominaciones, sentido y orden. Los tópicos establecidos son: contexto escolar, enseñanza del contenido de las ciencias sociales, metodología empleada en el aula y conducción de la clase. Sobre dichos tópicos, se seleccionan algunos fragmentos, como ejemplos de decisiones pedagógicas y de relaciones entre conocimiento y acción expresados por los futuros licenciados (Tabla 4).

TABLA 4. Ejemplos tópicos en la narrativa

\begin{tabular}{|c|c|}
\hline Tópico contexto escolar & $\begin{array}{l}\text { Visiones convencionales de características socio económicas y culturales del grado } \\
\text { en el que trabajan los practicantes y su influencia en el proceso de enseñanza }\end{array}$ \\
\hline Fragmento de la narrativa & Visión participante investigación \\
\hline $\begin{array}{l}{[. . .] \text { en el afán de cumplirle }} \\
\text { al colegio, a la profesora } \\
\text { titular del curso y por } \\
\text { supuesto al director de } \\
\text { práctica, se le termina } \\
\text { olvidando a uno eso que ha } \\
\text { diagnosticado y que hace } \\
\text { parte de la caracterización } \\
\text { del contexto escolar que nos } \\
\text { piden en la licenciatura. } \\
\text { (Diario de campo/Valentina } \\
\text { y Mariana) }\end{array}$ & 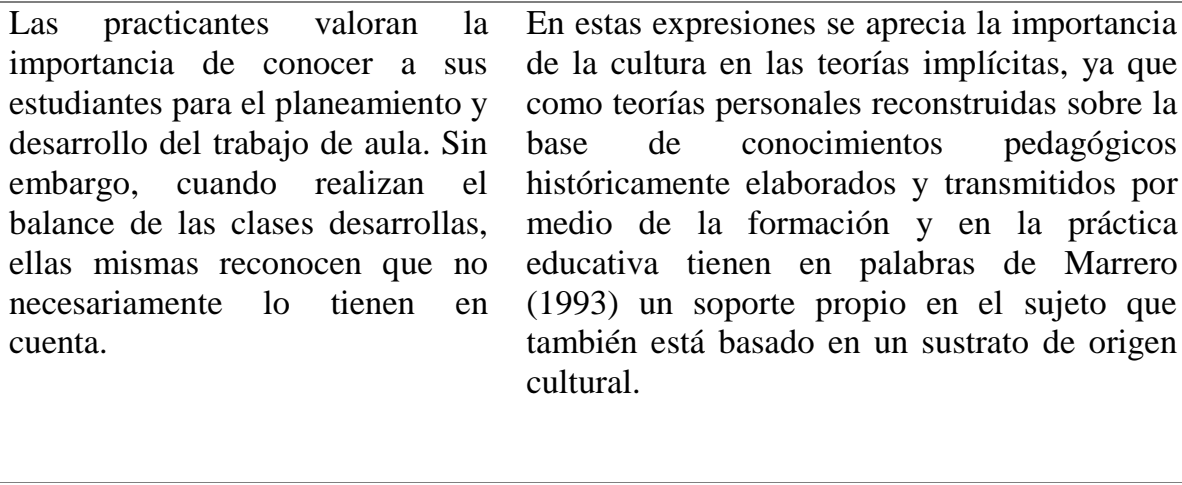 \\
\hline $\begin{array}{c}\text { Tópico enseñanza del } \\
\text { contenido de las ciencias } \\
\text { sociales }\end{array}$ & $\begin{array}{l}\text { Contenidos programáticos asignados a lo } \\
\text { asignatura y de área }\end{array}$ \\
\hline Fragmento de la narrativa & Visión participante investigación \\
\hline $\begin{array}{l}{[\ldots] \text { para ser un buen }} \\
\text { profesor hay que conocer } \\
\text { muy bien la disciplina que se } \\
\text { va a enseñar (Diario de } \\
\text { campo/Alejandro). }\end{array}$ & $\begin{array}{ll}\text { Los planteamientos que realizan } & \text { Esto corresponde con rasgos del pensamiento } \\
\text { los practicantes son recurrentes } & \text { espontáneo referido por Viveiro y De Senzi } \\
\text { frente a la importancia de conocer } & (2013) \text { y Arias y Álvarez (2013) en el cual } \\
\text { el contenido disciplinar de las } & \text { tiene un mayor privilegio la solvencia en el } \\
\text { ciencias sociales para enseñarlas } & \text { saber específico considerado por los } \\
\text { bien en la escuela. } & \begin{array}{l}\text { practicantes como suficiente, para un acertado } \\
\text { desenvolvimiento pedagógico. }\end{array}\end{array}$ \\
\hline
\end{tabular}




\begin{tabular}{|c|c|}
\hline $\begin{array}{l}\text { Tópico metodología } \\
\text { empleada en el aula }\end{array}$ & $\begin{array}{l}\text { Formas de trabajo que emplean los practicantes en el aula, quienes combinan } \\
\text { modalidades magistrales e innovadoras }\end{array}$ \\
\hline Fragmento de la narrativa & Visión participante investigación \\
\hline $\begin{array}{l}\text { [...] cuando se observa el } \\
\text { desempeño de estos } \\
\text { estudiantes que estoy } \\
\text { orientando, se ve la } \\
\text { predisposición personal que } \\
\text { tienen en su forma de } \\
\text { enseñar, } 4 \text { con } r a \\
\text { reproducción natural de } \\
\text { modelos aprendidos de otros } \\
\text { profesores y la definición } \\
\text { poco a poco de un estilo } \\
\text { propio, que se concretará } \\
\text { realmente en su desempeño } \\
\text { profesional (Diario de } \\
\text { campo/Samuel director de } \\
\text { práctica). }\end{array}$ & $\begin{array}{ll}\text { El director de práctica insiste que } & \text { Se reconoce la importancia del pensamiento } \\
\text { es un indicador del estilo de } & \text { espontáneo de los futuros licenciados, en el } \\
\text { enseñanza que cada practicante } & \text { cual como afirma Imbernon (2007) los } \\
\text { empieza a perfilar. En dicho } & \text { modelos pedagógicos con los cuales } \\
\text { estilo, se observan algunas } & \text { interactúan en su aprendizaje se extienden al } \\
\text { tendencias representativas en la ejercicio de la profesión y se convierten } \\
\text { planificación, ejecución } \mathrm{y} \text { incluso de manera involuntaria en referentes } \\
\text { evaluación del aprendizaje. }\end{array}$ \\
\hline Tópico manejo de la clase & $\begin{array}{l}\text { Habilidad del docente para alcanzar los objetivos definidos en su planifi } \\
\text { diaria, lo cual le permite desenvolverse apropiadamente en su actividad cot } \\
\text { tanto en situaciones previstas como imprevistas que se le puedan presentar }\end{array}$ \\
\hline Fragmento de la narrativa & Visión participante investigación \\
\hline $\begin{array}{l}\text { [...] por favor necesito que } \\
\text { nos escuchemos, el próximo } \\
\text { que siga hablando lo cambio } \\
\text { de puesto, porque yo no } \\
\text { estoy aquí hablándole a las } \\
\text { paredes ni nada de eso, listo } \\
\text { niños } \\
\text { (Valentina/V.MIV_0233.M } \\
\text { OV.27:3). }\end{array}$ & $\begin{array}{ll}\text { Parece que el aula se convierte en } & \text { Lo mencionado, se vincula igualmente con lo } \\
\text { un campo de batalla o por lo } & \text { que dice Finkel (2000) sobre que el modelo } \\
\text { menos así lo anuncian los } & \text { natural de dar clase es narrar, en el sentido de } \\
\text { practicantes, en que todo el } & \text { contar clara y cuidadosamente a los } \\
\text { tiempo se miden fuerzas entre el } & \text { estudiantes algo que ellos desconocen } \\
\text { profesor y los estudiantes para previamente. La transmisión de los } \\
\text { mantener el control de la clase. } & \text { conocimientos continúa diciendo el autor, al } \\
& \text { papel y el examen como instrumento de } \\
& \text { confirmación y control de que se ha producido } \\
& \text { dicha transmisión. }\end{array}$ \\
\hline
\end{tabular}

Fuente: Elaboración propia

\subsection{Anuncios en los conglomerados de relevancia}

La interpretación de la narrativa se basa en la metodología de conglomerados de relevancia, fundamentada en la idea de valor planteada por Molina (2005, 2007a, 2007b), para identificar la importancia, legitimidad y conveniencia de aquello que los profesores en formación inicial, consideran significativo en los relatos que componen la narrativa y reflejan los valores diferenciados que otorgan a su práctica pedagógica.

En este sentido, los criterios de valor no se establecen como ejes predefinidos dada la evolución que se produce en la lectura de los datos, a partir del proceso de construcción de la narrativa. Por ello, estos criterios adoptan una connotación de ejes abiertos que se proponen y consolidan posterior a la validación de esta. Las caracterizaciones de criterios de valor desarrollados por Molina (2002, 2012); Venegas (2015); Pedreros (2015); Valderrama (2016) y Peñaloza (2017), orientan la organización y jerarquización de estos en la investigación, a partir de la fuerza de las expresiones que utilizan los profesores en formación inicial durante sus clases.

La definición de los criterios de valor tiene en cuenta para su validación, un análisis de coocurrencias sobre relaciones representativas a nivel semántico entre códigos para realizar agrupaciones de sentido. Dichas relaciones se presentan a los participantes, con la selección de 
enunciados asociados a estas relaciones, para su ponderación. Este proceso permite definir como criterios de valor: saber disciplinar (S.disc.), ejercicio docente (E.doc.), desempeño de aula (D.aula.) y saber experiencial (S.exp.).

El criterio saber disciplinar (S.disc.) privilegia los saberes específicos de la disciplina objeto de enseñanza. Estos pueden tener matices hacia la transposición didáctica y/o la consideración de disciplinas escolares que circulan en el aula de clase. El criterio ejercicio docente (E.doc.) enfatiza en los procesos centrales de su profesión: la enseñanza y el aprendizaje de los estudiantes, relacionados con el saber propio del docente y las habilidades pedagógicas que emplea para resolver situaciones y dilemas escolares.

Por su parte, el criterio desempeño de aula (D.aula.) prioriza la manera como el docente ejerce autoridad en las formas de trabajo empleadas en clase, en las maneras de abordar el contenido, así como en procedimientos, actividades y formas de evaluación desarrollados por este; y el criterio saber experiencial (S.exp.) da mayor importancia a saberes prácticos específicos que dependen de su ajuste a las funciones, situaciones y problemas característicos del trabajo docente.

La jerarquización de los criterios no está dada sólo por el número de expresiones en cada uno, sino también por la importancia que tiene en el proceso de práctica pedagógica para el profesor en formación inicial. Lo anterior, se realiza elaborando jerarquías de dependencia de cada criterio de valor para apreciar su organización.

Así mismo, para dar coherencia a los conglomerados de relevancia definidos, estos se aplican a los tópicos (Top) destacados por los practicantes en la narrativa: contexto escolar (Top1 ce), enseñanza del contenido de las ciencias específicas (Top2 ecce), metodología empleada en el aula (Top3 mea) y conducción de la clase (Top4 cdc), para reconocer las jerarquías de dependencia de los criterios de valor en torno a los mismos.

Es importante mencionar, que en ellos ya hay un principio de selección y jerarquización por parte de los futuros licenciados, otorgando un valor especial a los aspectos mencionados. De igual manera, es necesario señalar que la organización de los tópicos frente al conglomerado de relevancia, también se presenta a los practicantes como un mecanismo de validación, produciéndose algunos cambios cuyo resultado final se plantea en la interpretación realizada. Las jerarquías indicadas en los conglomerados de relevancia implican los criterios S.disc. (saber disciplinar), E.doc. (ejercicio docente), D.aula. (desempeño de aula) y S.exp. (saber experiencial) en los tópicos (Top) 1, 2, 3 y 4, cuyas relaciones se describen en la tabla 5.

En sentido general, existe una mayor determinación del criterio de saber disciplinar (S.disc.) pues este orienta una serie de actuaciones y prácticas particulares que se vinculan de modo directo o indirecto con los demás criterios (D.aula., E.doc., S.exp.), que facilitan el reconocimiento de los elementos considerados importantes por los practicantes en su práctica pedagógica. Es necesario tener en cuenta, que el criterio de saber experiencial (S.exp.), a pesar de la importancia que le otorgan los practicantes en la oralidad, en su ejercicio docente aparece en un rango de presencia bajo a excepción de Top1 que es definido por éste.

En el conglomerado de relevancia en el Top1 (ce), se aprecian como criterios centrales el saber experiencial (S.exp.) y desempeño de aula (D.aula.) referido en este caso al contenido a enseñar. El criterio central de saber experiencial (S.exp.) estima un aspecto relacionado con la historia escolar de los practicantes y la derivación de dependencia del desempeño de aula (D.aula.), se vincula a la percepción que tienen de la clase y de los grupos de estudiantes a su cargo. Por otra parte, tiene en cuenta según Mateos $(2008,2009)$ el valor de la imagen que guardan los sujetos en su memoria sobre la institución escolar, la cual es el resultado de experiencias significativas (positivas o negativas) que hayan tenido durante su estancia en la misma. 
TABLA 5. Conglomerados de relevancias licenciatura en ciencias sociales

\begin{tabular}{|c|c|c|c|c|}
\hline Nombre & Tópico & Jerarquía configurada & \multicolumn{2}{|l|}{ Esquema } \\
\hline $\begin{array}{l}\text { Jerarquía de } \\
\text { dependencia } \\
\text { uno - JD1 }\end{array}$ & \begin{tabular}{l}
\multicolumn{3}{c}{ Top. 1: } \\
Integrado por los \\
criterios de valor: \\
S.exp., D.aula., \\
S.disc. y E.doc.
\end{tabular} & $\begin{array}{l}\text { Criterios } \\
\text { * S.exp. y D.aula. con una } \\
\text { relación horizontal; } \\
\text { * S.exp. y D.aula. con una } \\
\text { relación vertical con S.disc.; } \\
\text { *S.disc. con una relación } \\
\text { vertical con E.doc. }\end{array}$ & $\begin{array}{c}\downarrow \\
\downarrow \\
\text { E.disc. } \\
\text { E.doc. }\end{array}$ & $1 \mathrm{a}$. \\
\hline $\begin{array}{l}\text { Jerarquía de } \\
\text { dependencia } \\
\text { dos - JD2 }\end{array}$ & $\begin{array}{l}\text { Top. 2: } \\
\text { Integrado por los } \\
\text { criterios de valor: } \\
\text { S.disc., } \\
\text { D.aula. y S. exp. }\end{array}$ & $\begin{array}{l}\text { Criterios } \\
* \text { S. disc. con una relación } \\
\text { vertical con E.doc., D.aula. y } \\
\text { S.exp. }\end{array}$ & Edoc. & S.exp. \\
\hline $\begin{array}{l}\text { Jerarquía de } \\
\text { dependencia } \\
\text { tres - JD3 }\end{array}$ & $\begin{array}{l}\text { Top. 3: } \\
\text { Integrado por los } \\
\text { criterios de valor: } \\
\text { S.disc., } \\
\text { E.doc. y S. exp. }\end{array}$ & $\begin{array}{l}\text { Criterios } \\
* \text { S. disc. y D.aula. con una } \\
\text { relación horizonal; } \\
* \text { S. disc. y D.aula con una } \\
\text { relación vertical con E.doc.; } \\
* \text { E.doc. con una relación } \\
\text { vertical S.exp. }\end{array}$ & $\frac{\Downarrow}{\text { S.exp. }}$ & ula. \\
\hline $\begin{array}{l}\text { Jerarquía de } \\
\text { dependencia } \\
\text { cuatro-JD4 }\end{array}$ & $\begin{array}{l}\text { Top. 4: } \\
\text { Integrado por los } \\
\text { criterios de valor: } \\
\text { D.aula., } \\
\text { S.disc. y } \\
\text { S.exp }\end{array}$ & $\begin{array}{l}\text { Criterios } \\
\text { *D.aula. y E.doc. con una } \\
\text { relación horizontal; } \\
\text { *D.aula. y E.doc. con una } \\
\text { relación vertical S. disc. y } \\
\text { S.exp.; } \\
\text { *S.disc. con una relación } \\
\text { horizontal con S.exp. }\end{array}$ & $\begin{array}{l}\text { D.alina. }< \\
\sqrt{\text { S.disc. }}<\end{array}$ & $\frac{}{\exp .}$ \\
\hline
\end{tabular}

Fuente: Elaboración propia

El desempeño de aula (D.aula.) por su parte, se relaciona desde las palabras de los practicantes con las características particulares del escenario familiar y socioeconómico de sus estudiantes, para tenerlo en cuenta en los procesos de enseñanza, que Mateos $(2008,2009)$ resalta en investigaciones que indagan sobre el papel relevante del marco social y cultural en la conformación de experiencias, valores y/o expectativas que se forman hacia la institución educativa.

Este componente es reiterado en los enunciados de los practicantes en la narrativa, sin embargo, las acciones observadas muestran el poco impacto que tienen en la enseñanza de las ciencias sociales. Esto muestra, que una es la importancia declarada al respecto y otra la ejercida efectivamente. No obstante, es claro que esta valoración significativa da cuenta de una manera de abordar el ejercicio docente en aula.

En este tópico se aprecia la distancia entre teoría y práctica, que puede visibilizarse de manera diferenciada en los modelos de formación inicial tradicional, instrumental y/o alternativo planteados por (Porlán et al., 1997). Al respecto, los practicantes consideran que no desarrollan sus clases con un modelo de formación exclusivo y se autodefinen de manera recurrente como constructivistas, estableciendo una negativa categórica sobre el modelo de formación tradicional. 
El conglomerado de relevancia en el tópico enseñanza del contenido de ciencias sociales (Top2 eccs), da un mayor valor a los criterios de saber disciplinar (S.disc.) y ejercicio docente (E.doc.), en los cuales se hace más evidente la relación de la práctica con los modelos antes mencionados y que Barrón (2015) caracteriza diciendo que el énfasis del saber disciplinar y la transmisión del mismo, la división entre teoría y práctica y la imitación como una manera de conseguir profesionalidad, es lo central en el modelo de formación inicial tradicional.

Se podría afirmar entonces, que el interés central para los futuros docentes es desempeñar bien su labor de enseñanza, para lo cual es fundamental para ellos tener un conocimiento profundo de la disciplina. Esto, además de ser considerado una habilidad pedagógica esencial, también le confiere seguridad y autoridad al practicante en el aula.

En este punto, la perspectiva de transposición didáctica expuesta en la narrativa, permite según Thémines (2009) analizar los cambios de un saber de origen erudito cuando la escuela se apodera de él. Las expresiones de los practicantes enfatizan igualmente, en la importancia del conocimiento del saber disciplinar para enseñar; pero también hacen alusión al manejo de contenidos, ejercicios, finalidades, procedimientos de motivación, constituyentes de la disciplina escolar planteada por Chervel (1988).

Finalmente, en el conglomerado de relevancia del tópico de manejo de la clase (Top4 mc), se aprecia como criterio central el desempeño en el aula (D.aula.), del cual depende el ejercicio docente (E.doc.). Este desempeño ya no se plantea desde lo metodológico sino desde lo actitudinal, referido al tema de la posición y manejo de autoridad del docente frente al grupo de estudiantes con el que trabaja. Las referencias de los profesores en formación inicial sugieren una tensión permanente entre autoritarismo y ausencia de autoridad en las acciones que desarrollan en aula. Muchas situaciones escolares cotidianas ponen de manifiesto de acuerdo con Tallone (2011), un cambio respecto al consenso social que otorgaba al docente de manera incuestionable, la función de autoridad. Por el contrario, se asiste con más frecuencia a que la autoridad legítima del docente se ve sometida a un constante ejercicio de legitimación, es decir, su autoridad vista desde normas y valores aceptados en conjunto, requieren cada vez más una validación y reconocimiento social.

Por ello, los practicantes consideran un desempeño de aula eficiente y eficaz, aquel en el que tienen el control de la situación, en el que regulan el ritmo y tiempos de los procesos de enseñanza y aprendizaje, que pueden contemplar o no las posibilidades del estudiante, haciendo valer su autoridad tanto académica como funcional.

\section{CONCLUSIONES FINALES}

Los resultados de investigación aportan en el acercamiento a la práctica pedagógica desde los territorios epistémicos descubiertos, en las convergencias entre conocimiento y acción de los practicantes. En ellas, la experiencia personal y escolar, el conocimiento personal docente, las teorías implícitas y los aprendizajes formales a nivel pedagógico y disciplinar, juegan un papel fundamental con su carácter integrador para introducir cambios en los desarrollo de la práctica docente en la escuela.

Así mismo, se reconoce la existencia de un límite y no una frontera entre teoría y práctica en un territorio epistémico, en el que estos elementos aparecen en la narrativa como complementarios, pero que en el ejercicio de aula son antagónicos. Lo que supone una reflexión y especialmente una materialización más real, para resignificar su presencia, tanto en la propuesta formativa de la licenciatura, como en la organización, orientación y asesoría de la intervención escolar.

Este es otro aspecto novedoso, que reitera el valor de apreciar la práctica pedagógica como un espacio cognitivo-axiológico, en el que estas dimensiones se exploren de manera intencionada en practicantes, directores de práctica y docentes de las instituciones escolares; para reflexionar sobre ellas y aplicarlas en la práctica docente; pensando en un ejercicio más digno y no reproductivo de guiones y rutinas por parte de los futuros licenciados. Esto sitúa la discusión, no en la práctica 
pedagógica como sinónimo de actividades y acciones, sino como una praxis que se vincula en la formación, en tanto reflexión consciente del ejercicio de la docencia como afirma García (2000).

Igualmente, se descubren territorialidades pedagógicas, como espacios demarcados de la acción reflexiva del docente en formación, en las cuales se reconocen áreas de inter conocimiento desde la propuesta De Sousa Santos (2010), que reitera la presencia de límites entre lo teórico y lo práctico en la enseñanza, la expression de fronteras entre actitudes transmisionistas y constructivistas sobre el conocimiento y una territorialiadad asociada con la formación docente tradicional o alternativa, cuya síntesis se presenta a continuación.

Límites entre lo teórico y lo práctico en la enseñanza: es un territorio que muestra una tensión entre una ciencia enseñada desde una perspectiva disciplinar y teórica y una ciencia enseñada desde una perspectiva empleada y aplicada. En la primera tensión, esta se aprecia por ejemplo en las actuaciones de los practicantes cuando explican un contenido, apoyados en la transposición didáctica o en la perspectiva de disciplina escolar propiamente dicha. En la segunda tensión, se identifica el énfasis que da el practicante a distintas actividades para desarrollar un contenido, en el que centra la atención en experiencias cotidianas como única fuente de aprendizaje o en la lectura del saber teórico a situaciones concretas para ser aplicados en ellas.

En la intersección se encuentra el protagonismo que sigue manteniendo el profesor en formación inicial en la orientación dada a la clase, con la introducción de estrategias que invierten el rol pasivo de los estudiantes, fundamentalmente en términos de su participación para conocer preconcepciones, reconocer comprensiones y verificar aprendizajes, más que en una relación distinta con el saber que en la práctica pedagógica sigue siendo de carácter canónico y decimonónico frente a las ciencias sociales, es decir, universal e incuestionable sobre lo que plantea el docente.

Fronteras entre actitudes transmisionistas y constructictivistas sobre el conocimiento: es un territorio que muestra tensiones entre formas de asumir la relación con el conocimiento y con los desarrollos de aprendizaje que se esperan en los estudiantes. Por ello, en la intersección del territorio se generan encuentros, entre un proceso educativo dirigido por el practicante centrado en su autoridad como afirma Marrero (1988) bien sea de carácter físico moral y otro asumido de manera activa por el estudiante en ejercicios problematizadores de la realidad, para descubrir y/o construir conocimiento.

Este territorio pone de manifiesto la convergencia entre teorías explícitas e implícitas, es decir, entre modelos académicos aprendidos y experiencias personales y sociales a nivel escolar que inciden en la toma de decisiones de situaciones particulares de la práctica pedagógica pero también de la práctica profesional en conjunto.

Formación docente tradicional o alternativa: es una territorialidad que sugiere la organización de dos espacios pedagógicamente diferenciados, producto de un ejercicio de poder, que marca tendencias entre modelos formativos convencionales y activos. En ellos, las nociones de monodisciplina en uno e interdisciplina en otro, encontradas por Marrero (1988) en estudios de creencias en profesores universitarios, establece formas de acercamiento al saber y actitudes diferenciadas de acuerdo con las características que se ponen en juego en estos modelos formativos en el desempeño de aula por parte del profesor en formación.

Este territorio tiene su intersección en actuaciones conductistas, eficientistas, constructivistas y/o críticas por parte de los practicantes, que en las situaciones cotidianas de aula se evidencian permanencias, transformaciones, dilemas y/o incertidumbres que sugieren la necesidad de aumentar la reflexión pedagógica al respecto, en la escuela y la universidad como instancias de formación docente, en las cuales el tema no es ajeno pero de acuerdo con las expresiones de los practicantes se queda en el umbral más de la conceptualización que de la acción.

Se podría concluir, que algunos de los aportes de la investigación están en la reflexión teórico-práctica sobre las biografías personales y escolares y teorías implícitas de los futuros docentes, para analizar concepciones, creencias y acciones sobre su acto educativo; el 
reconocimiento de las trayectorias de actuación de los docentes en formación, como aporte a la configuración de su episteme profesional y la reflexión de la práctica pedagógica desde las intersecciones entre conocimientos y acciones en los territorios epistémicos identificados.

\section{Referencias}

Ameigeiras, A. (2009). El abordaje etnográfico en la investigación social. En I. Vasilachis (Coord.), Estrategias de investigación cualitativa. Barcelona: Gedisa Editorial, S.A.

Arias, A., Álvarez, M. y Álvarez, F. (2013). Concepciones del profesorado en formación inicial sobre los roles docentes y discentes en el aprendizaje de las ciencias en la educación infantil y primaria. Enseñanza de las ciencias. Revista de investigación y experiencias didácticas, número extra, 194-201.

Barrón, C. (2015). Concepciones epistemológicas y práctica docente. Una revisión. REDU Revista de docencia universitaria, $\mathrm{v} .13, \mathrm{n}^{\circ} 1,36-56$.

Claxton, C. y Murrell, P. (1987). Learning Styles: Implications for Improving Educational Practices. Washintong: ASHE-ERIC Higher Education Report No. 4.

Clark, C. y Peterson, P. (1990). Procesos de pensamiento de los docentes. En M. Witrock (Comp.), La investigación de la enseñanza Vol III. Barcelona: Paidós.

Chevallard, Y. (1991). La transposición didáctica: del saber sabio al saber enseñado. Argentina: Aique Grupo Editor S.A.

Chervel, A. (1988). L'histoire des disciplines scolaires. Histoire de l'éducation, 38, 59-119.

Cole, M. y Engrestrom, Y. 2001. Enfoque histórico - cultural de la cognición distribuida. Cogniciones distribuidas. Consideraciones psicológicas y educativas. Buenos Aires: Amorrortu.

Creswell, J. (1994). The use of a theory. Research Desing. Qualitative y Quantitative Approaches. Thousand Oaks: Sage Publications.

De Longui, A. (2000). El discurso del profesor y el alumno: análisis didáctico en clases de ciencias. Enseñanza de las ciencias, 18(2), 201-216.

De Sousa Santos, B. (2010). Descolonizar el saber, reinventar el poder. Uruguay: Ediciones Trilce.

Elbaz, F. (1983). Teacher thinking: a study of practical knwolegde. New York: Nichols Publishing Company.

Elbaz, F. (1991). Research o teachers' knwolegde: The evolution of a discourse. Journal o Curriculum Studies. 23(1), 1-19.

Fenstermacher, G. (1994). The knower and the known: The nature of knowledge in research on teaching. Review of Research in Education, 20, 3-56.

Finkel, D. (2000). Teaching with your mouth shut. Butterworth Heinemann Estados Unidos: Boynton/Cook.

García, F. (2000). Un modelo didáctico alternativo para transformar la educación el modelo de investigación en la escuela. Scripta Nova: Revista electrónica de geografía y ciencias sociales, 4(64), 1-24.

Hammersley, M. y Atkinson, P. (1994). Etnografía. Barcelona: Paidós.

Hatano, G. y Inagaki, K. (1994). Young children's naive theory of biology. Cognition, 50, 171-188.

Imbernón, F. (2007). La formación y el desarrollo profesional. Hacia una nueva cultura profesional. Barcelona: Editorial Grao de IRIF, SL.

Jessup, M., Pulido, R. y Fernández, M. (2013). Formación docente y educación para el desarrollo humano integral. Revista Internacional Magisterio. Educación y Pedagogía, 65, 16-18.

Keil, F. (1992). The origin of an autonomous biology. En M. Gunnar y M. Maratsos (Eds.), Modularity and constraints in language and cognition. The Minnesota Symposia on Child Psychology. Erlbaum: Hillsdale. 
Marrero, J. (1988). Teorías implícitas y planificación del profesor (Tesis doctoral). Universidad de La Laguna, España.

Marrero, J. (1993). Las teorías implícitas del profesorado: vínculo entre la cultura y la práctica de la enseñanza. En M. Rodrigo, A. Rodríguez y J. Marrero (Coords.), Las teorías implícitas. Una aproximación al conocimiento cotidiano. Madrid: Aprendizaje Visor.

Mateos, T. (2008/2009). La percepción del contexto escolar. Una imagen construida a partir de las experiencias de los alumnos. Cuestiones Pedagógicas, 19, 285-300.

Maxwell, J. (1996). Qualitative research design. An Interactive Approach. California: Sage Publications.

Mellado, J. y González, B. (2000). La formación inicial del profesorado de ciencias. En F. Perales y P. Cañal (Eds.), Didáctica de las ciencias experimentales. Teoría y práctica de la enseñanza de las ciencias. España: Editorial Marfil S.A.

Molina, A. (2002). Conglomerado de relevancias y formación científica de niños, niñas y jóvenes. Centro de Investigaciones y Desarrollo Científico, 4(1), 187-200.

Molina, A. (2005). El "otro" en la constitución de identidades culturales. En C. Piedrahita y E. Paredes (Eds.), Cultura política, identidades y nueva ciudadanía, 2, 139-169.

Molina, A. (2007a). Relaciones entre contexto cultural y explicaciones infantiles acerca del fenómeno de las adaptaciones vegetales. Nodos y Nudos, 3(23), 76-87.

Molina, A. (2007b). Analogía, pensamiento científico infantil y revalorización de las teleologías y el antropomorfismo. Tecne Episteme y Didaxis, número extra, 88-107.

Molina, A. (2012). Contribuciones metodológicas para el estudio de las relaciones entre contexto cultural e ideas sobre la naturaleza de niños y niñas. En A. Molina (Ed.), Énfasis libros de los énfasis del Doctorado Interinstitucional en Educación. Algunas aproximaciones a la investigación en educación en enseñanza de las Ciencias Naturales en América Latina. Bogotá: Javegraf.

Nates, B. (2011). La territorialización del conocimiento. Categorías y clasificaciones culturales como ejercicios antropológicos. Barcelona: Anthropos Editorial.

Neiman, G. y Quaranta, G. (2009). Los estudios de caso en la investigación sociológica. En I. Vasilachis (Coord.). Estrategias de investigación cualitativa. Barcelona: Gedisa Editorial, S.A.

Pedreros, R. (2015). Perfil conceptual de calor y conglomerados de relevancias en comunidades culturalmente diferenciadas (Tesis doctoral). Universidad Distrital Francisco José de Caldas, Bogotá.

Peñaloza, G. (2017). Relaciones ciencia - religión y enseñanza de la evolución. Estudio de casos con profesores de biología de educación básica secundaria en Colombia. (Tesis doctoral). Universidad Distrital Francisco José de Caldas - Universidad de Bahía, Bogotá.

Porlán, R. y Martín, J. (1994). El diario del profesor. Un recurso para la investigación en el aula. Sevilla: Editorial DIADA.

Porlán, R. Rivero, A. y Martín del Pozo, R. (1997). Conocimiento profesional y epistemología de los profesores, I: Teoría, métodos e instrumentos. Enseñanza de las ciencias, 15(2), 155-171. Recuperado de http://www.raco.cat/index.php/ensenanza/article/viewFile/21488/93522 [28 de febrero de 2014].

Rando, W. y Menges, R. (1991). How practice is shaped by personal theories. New Directions for Teaching and Learning, 45, 7-14.

Rodríguez, L. 2015. Aproximaciones a la conceptualización de territorio epistémico. En W. Mora (Comp.), Énfasis Educación en ciencias: experiencias investigativas en el contexto de la didáctica, la historia, la filosofía y la cultura. Bogotá: Fondo de Publicaciones Universidad Distrital Francisco José de Caldas.

Rodrigo, M., Rodríguez, A. y Marrero, J. (1993). Las teorías implícitas: Una aproximación al conocimiento cotidiano. Madrid: Visor. 
Rodríguez, D. y Vallderiola, J. (2009). Metodología de la investigación. Barcelona: Universitat Oberta de Catalunya.

Stake, R. (1999). Investigación con estudio de casos. Madrid: Ediciones Morata S.A.

Smith, L. (1979). An evolving logic of participant observation, educational etnography, and other case studies. Review of Research in Education, 6, 316-377.

Tallone, A. (2011). Las transformaciones de la autoridad docente, en busca de una nueva legitimidad. Revista de Educación, número extra, 115-135.

Tenti Fanfani, E. (2010). Viejas y nuevas formas de autoridad docente. Recuperado de http://www.revistatodavia.com.ar/todavia07/notas/tenti/txttenti.html.

Thémines, J. (2009). Investigación y formación del profesorado en didáctica de la geografía: posibilidades e implicaciones de algunas investigaciones en las prácticas docentes en Francia. Enseñanza de las ciencias sociales, 8, 13-23.

Valderrama, D. (2016). Diálogo entre conhecimentos científicos escolares e tradicionais em aulas de ciências naturais: intervenção e pesquisa na comunidade de Taganga (MagdalenaColômbia) (Tesis doctoral). Universidade Federal de Bahía, Salvador (Brasil)

Venegas, A. (2015). Diversidad cultural, enseñanza de las ciencias e ideas de naturaleza de niños y niñas (Tesis doctoral). Universidad Distrital Francisco José de Caldas, Bogotá (Colombia).

Vivero, A. y De Senzi, M. (2013). A formação de professores para o ensino de ciências nos anos inicias da escolarização: reflexões e perspectiva para exploração da natureza da ciência. Enseñanza de las ciencias. Revista de investigación y experiencias didácticas, número extra, 3732-3736.

CÓMO CITAR ESTE ARTÍCULO

Rodríguez Pizzinato, L. A. (2018). Territorios epistémicos en la práctica pedagógica en ciencias sociales. Didáctica de las ciencias experimentales y sociales, 35, 17-32. DOI: 10.7203/DCES.35.12913. 